\title{
IDENTIDAD NACIONAL Y TELEVISIÓN: POLÍTICAS DE COMUNICACIÓN Y CULTURA EN NICARAGUA EN LA DÉCADA DE 80
} (comparaciones y aproximaciones con la experiencia brasileña)

Felipe Canova Gonçalves / Elen Cristina Geraldes

\section{Resumen}

En este artículo se discuten las políticas de comunicación y cultura de Nicaragua en la década de 1980, cuando la televisión fue elegida como un medio para la construcción de una identidad nacional. El caso de Nicaragua, poco conocido en Brasil, puede integrarse en un esfuerzo por comprender el complejo escenario de las políticas de comunicación en América Latina. El aporte teórico fue dado por el concepto de identidad, entendido como un mecanismo de resistencia y de lucha contra los valores hegemónicos y como aporte para un proyecto nacional. Metodológicamente, se ha utilizado la investigación bibliográfica y el método histórico.

Palabras claves: políticas de comunicación, identidad, Estado, televisión.

\begin{abstract}
This paper discuss Nicaraguan communicational and cultural policies of 1980's decade, when television was selected as a media to build national identity. Nicaragua's case, little known in Brazil, can be integrated in an effort to understand the complexity of communications policies in Latin America. Theoretical contributions was given by identity concept, understood as resistance and fight mechanism against hegemonic values, as well as contribution to a national project. Literature review and historic method has been used to carry out this research.
\end{abstract}

Keywords: Communication policies, Identity, Television, Estate Government.

L as discusiones rectoras de las políticas públicas en el ámbito de la cultura en América Latina, tradicionalmente hacen referencia a cuestiones tales como la identidad nacional y la diversidad cultural dentro de un papel unificador del Estado. Un ejemplo reciente de Brasil es el Plan Nacional de Cultura, sancionado por el Presidente Luiz Inácio Lula da Silva en el año 2010, el cual tiene como primer objetivo, entre los dieciséis establecido, la necesidad de "reconocer y valorar la diversidad cultural, étnica y regional brasileña" (BRASIL, 2012, p. 159).

Reflexionar sobre la cultura nacional históricamente ha sido tomar una posición política sobre las orientaciones de la nación y el papel del Estado. Como dice Ortiz: "No es casualidad que la cuestión de la identidad está estrechamente relacionada al problema de la cultura popular y el Estado; en última instancia, hablar en la cultura brasileña es discutir el destino político de un país" (1994, p. 13). 
En la actualidad la discusión sobre el tema de las políticas de comunicación de América Latina asume otras perspectivas. La existencia de un consenso hegemónico sobre el rol del mercado como regulador de la dinámica de la comunicación y la cultura - que tiene su origen en la formación de las industrias culturales de nuestros países y de su consolidación en la reanudación de ideas liberales en la década de 1980 -, entra en conflicto con el surgimiento de gobiernos progresistas y de izquierda en la primera década de este siglo.

En medio de los cambios políticos, económicos y socioculturales provocados por el ascenso de estos gobiernos populares, progresistas y revolucionarios, se plantean entre otros retos, la consolidación de los nuevos paradigmas de la acción del Estado y las políticas públicas, en particular las políticas públicas de comunicación. Valores como "el pluralismo, la transparencia, la diversidad cultural, la creatividad, el desarrollo económico y tecnológico y el bienestar social" (BRITTOS; MIGUEL, 2009, p. 7), aclamados como claves por investigadores y activistas delas Políticas de Comunicación para la acción mediadora de los Estados contemporáneos se incorporan en este campo. Como paradoja se sabe que el concepto de identidad nacional pierde fuerza con la globalización y la aparición de un "multiculturalismo" que asume de manera rápida una lógica funcional a los intereses del capital.

En este artículo se reflexiona acerca de las relaciones existentes entre las políticas de comunicación y cultura y los conceptos de identidad nacional y diversidad cultural. Para ello problematizaremos sobre el papel del Estado como mediador de las políticas públicas, con una mirada en las políticas de comunicación y cultura creadas en Nicaragua durante los años 80, por medio del Sistema Sandinista de
Televisión como un elemento clave en la apropiación de las ideas de la identidad nacional y la diversidad cultural por medio de la comunicación estatal. El caso de Nicaragua, poco conocido en Brasil, puede integrarse en un esfuerzo por comprender el complejo escenario de las políticas de comunicación en América Latina.

El aporte teórico de esta reflexión se sustenta en el concepto de identidad. En la primera parte de este artículo consideramos su triple afiliación a los estudios de comunicación: la identidad como el multiculturalismo o la diversidad; identidad como la resistencia y lucha por la transformación social; y la identidad como un recurso central para el desarrollo de un proyecto nacional. Metodológicamente, utilizamos las técnicas de investigación bibliográfica y el hilo conductor del método histórico, que escapa a la linealidad e interpreta la historia desde un punto elegido de antemano.

\section{Identidad nacional y políticas de comunicación y cultura}

El Profesor Rento Ortiz, quien se ha involucrado de manera profunda en la discusión sobre la identidad nacional, los medios de comunicación y el proyecto de país forjado durante la dictadura militar en Brasil, destaca la doble naturaleza del concepto de la identidad en su génesis. En primer lugar "toda identidad se define en relación con algo que está fuera, es una diferencia" (2003, p. 7). Sin embargo, la observación de la diferencia en relación con el otro no es suficiente, se debe considerar una dimensión interna, es decir, "es necesario mostrar que podemos identificarnos" (2003, p. 7-8), lo que constituye un campo de confrontación cuando pensamos la cuestión nacional. 
Evidentemente, la idea de identidad nacional pasa por una construcción del "ser nacional" mediada por el Estado, evidente desde el punto de vista histórico en la intervención estatal en la cultura, sin importar si los regímenes son o no autoritarios. Barbalho afirma que tanto los países subdesarrollados de América Latina, como los países del primer mundo pasaron por "procesos de valoración de las identidades nacionales monolíticas" (2001, p. 2). Un ejemplo planteado por el autor, es la idea de "britanicidad", promovida por el gobierno conservador de la Thatcher en el Reino Unido de los 80, cuando apeló a la reanudación de los "valores propios", con el fin de atenuar y/o disolver los conflictos de clase en el pueblo británico. Esta práctica de carácter hegemónico, consistió en la "preparación de un discurso sobre la cultura nacional británica basada en el redescubrimiento y re-valoración de la edad de oro del Imperio Británico." (2001, p. 3).

Aunque Ortiz reconozca una de las opciones elegidas por el estado dictatorial brasileño (1964-1985), de una identidad nacional forjada por medio de la televisión, negando otras posibilidades y excluyendo la contribución de los grupos populares, no cree que este sea un concepto monolítico. La identidad para este autor, implica relaciones de poder, elecciones, intercambios, y en cada momento histórico se puede restablecer de forma más o menos plural. En este sentido, importan más la tensión y la fuerza de esos conflictos que ven la cultura como un espacio de afirmación o negación de uno mismo y el otro.

Una pregunta que surge de estos elementos en discusión es cómo relacionar la identidad nacional conla pluralidad delasidentidades propias de la contemporaneidad. Uno de los desafíos en la construcción de nuevas políticas públicas para la cultura y la comunicación es el cuestionamiento crítico de la diferencia, entendiéndola como una zona de conflicto. Barbalho establece que "una política cultural que se ocupa de las cuestiones de identidad tiene que tomar en cuenta las diferencias y relaciones de poder y la transitoriedad de las identidades y las diferencias" (2001, p. 5).

La incorporación de la idea de "multiculturalismo"1 se ha convertido en la actualidad, sea dentro del espacio académico o en la acción del Estado, como una alternativa a la fragmentación identitaria y a los límites de la construcción de una identidad nacional. El término diversidad asigna en este contexto tanto formaciones sociales radicalmente diferentes - "tribus indígenas, etnias, las civilizaciones y las naciones del pasado" - como grupos propios de la contemporaneidad tales como "movimiento de mujeres individuales, gays, negros, crisis de identidad, etc.", sin una distinción cualitativa entre las diferencias (ORTIZ, 1999, p. 81-82).

Reconocer que las diferencias se producen socialmente es un paso importante en esta discusión. A modo de ejemplo, recordamos que los temas comúnmente asignados a la diversidad y manifestaciones de lucha y resistencia como la emancipación de la mujer, los derechos de los pueblos indígenas, el movimiento negro, deben ser entendidos en sus particularidades, ya sea en su significado simbólico como en su sentido histórico. "Consolidar el sentido histórico de la diversidad cultural representa sumergirla en los intereses materiales y los conflictos sociales (el

1 "El respeto y la afirmación del derecho a la existencia y manifestación de las diversas expresiones culturales minoritarias de una sociedad determinada" (BARBALHO, 2001, p. 6). 
capitalismo, el socialismo, el colonialismo, la globalización). La diversidad se manifiesta en situaciones concretas." (ORTIZ, 1999, p. 83).

Por otro lado, la idea de la diversidad es fundamental para la reflexión sobre nuestra condición latinoamericana. La articulación cultura latinoamericana con unidad regional y esta relacionarla con nuestra diversidad, establece el camino trazado por muchos intelectuales que problematizaron sobre la formación de los pueblos latinoamericanos. Ángel Rama, crítico literario uruguayo, plantea la cuestión de la unidad como un proyecto real, impulsado por los intelectuales en base a razones convincentes y fuerzas unificadoras:

La mayoría de ellas radica en el pasado, habiendo modelado hondamente la vida de los pueblos: van desde una historia común a una común lengua y a similares modelos de comportamiento. Las otras son contemporáneas y compensan su minoridad con una alta potencialidad: responden a las pulsiones económicas y políticas universales que acarrean la expansión de las civilizaciones dominantes del planeta (2004, p. 57).

Con esta unidad de base planteada por Rama, se puede encontrar una "interior diversidad que es la definición más precisa del continente" (2004, p. 57). Esta diversidad, característica de la formación de América Latina se lleva a cabo tanto a nivel de las naciones y sus diversas iniciativas de integración, como por medio de la existencia de regiones culturales, que a menudo entran en conflicto con el concepto de unidad nacional. Para Ángel Rama, "si la unidad implica un sistema de diferenciaciones con las culturas extranjeras, (...) la macro regionalización implica una diferenciación interna por un correlativo sistema de oposiciones" (2004, p. 59), en los criterios establecidos por áreas como la antropología cultural, la economía y la historia.

\section{Identidad nacional y unidad: televisión en Brasil y Nicaragua}

En un momento particular de la historia de Brasil, el debate sobre la identidad nacional estuvo relacionado a la formación de una industria cultural nacional. Nos referimos al proyecto autoritario de la dictadura cívico-militar iniciada en 1964, cuando la política estatal centrada en el binomio integración-seguridad nacional apoyó la consolidación de un mercado de bienes culturales en el país, mediante el desarrollo de la televisión como un elemento clave.

Creo que lo que mejor caracteriza la aparición y consolidación de la industria cultural en Brasil es el desarrollo de la televisión. (...) Con la inversión del estado en el área de las telecomunicaciones, los grupos privados tenían la primera vez la oportunidad de hacer realidad sus objetivos de integración de los mercados. (ORTIZ, 1994, p. 128).

María Rita Kehl, en su esfuerzo de analizar la estructuración de TV Globo en ese período, plantea que el papel de la televisión era tomar la idea de la integración nacional - sueño político de los militares a nivel del imaginario, donde las imágenes transmitidas de manea simultánea, en "un país tan dividido como Brasil, contribuyen a transformarlo en una apariencia de nación, cuya población, unificada no como "pueblo", sino como espectadores, articula un lenguaje común a la misma sintaxis." (1986, p. 170). La aspiración a la entrada en la modernidad - expresada en las 
imágenes de televisión de un Brasil urbano, cosmopolita, industrial - representaba una voluntad que, para Ortiz, "en los países periféricos se articula estrechamente a la construcción de la identidad nacional" (1994, p. 34).

Televisión e integración nacional: la inclusión de las subculturas aisladas entre si y lejanas de los centros de producción cultural, en una visión clara del mundo, ordenada y unificada. Publicidad y la integración nacional: la inclusión de las subculturas aisladas entre si, etc. etc., en un orden de deseos, símbolos y hábitos propios de la sociedad de consumo. (KEHL, 1986, p. 170).

No obstante, se puede afirmar que la estructuración de la televisión en un determinado país, como política de Estado, y su relación con la identidad nacional no se consolida solamente mediante la creación de un mercado de bienes culturales. Un posible contrapunto a la formación de la televisión brasileña es la experiencia de Nicaragua, durante el período de la Revolución Popular Sandinista (19791990).

Un levantamiento popular en julio del 1979 derroca la dictadura de la familia Somoza perpetuada por más de 43 años, y lleva al poder al Frente Sandinista de Liberación Nacional (FSLN), conformando una Junta de Gobierno de Reconstrucción Nacional en la cual se incluyen otras fuerzas políticas progresistas que habían luchado por años contra la dictadura.. Desvastado el país por la guerra civil, se harán cambios estructurales urgentes, entre los que se cuenta el modelo de comunicación, que desde su conformación, según el investigador nicaragüense Rothschuh Villanueva siguió la decisiva influencia del modelo de EE.UU. donde los medios de comunicación estaban atados a los intereses inmediatos de las clases dominantes locales y del mercado (Villanueva, 1992).

Los principios del pluralismo político y la economía mixta, adoptados por los sandinistas, fueron centrales en la nueva conformación de los medios de comunicación. No existió - excepto en la televisión, diseñada como servicio público estatal - un sistema único de comunicación, sino una situación de competencia de los nuevos medios de comunicación del Estado con los que sobrevivieron del modelo somocista, muchos de los cuales se convertirían en opositores. Esta etapa de reestructuración de los medios de comunicación, que la investigadora Penélope O'Donnell define como "reconfiguración de la oferta cultural", se consolidó en cuatro puntos principales: "se reconfiguró de manera total el régimen de propiedad del sistema de medios de comunicación masiva en Nicaragua, su marco jurídico, la institución rectora y su orientación cultural" (1993, p. 226).

La creación del Ministerio de Cultura al comienzo de la revolución fue fundamental en la consolidación de una nueva organización de los medios de comunicación, ya que era la institución directamente responsable de ellos, que buscaba la creación de una amplia oferta cultural. Como objetivos generales de su trabajo, el documento "Un Programa de la Revolución: Ministerio de Cultura" señaló:

...neutralizar las influencias prevalecientes y heredadas del pasado a través del rescate y de la preservación de los valores propios que conforman la identidad nacional nicaragüense, que es latinoamericana, y desarrollar, basados en nuestros propios valores, una cultura democrática y antiimperialista, por medio de la cual el 
pueblo se convierta en creador de la cultura y en su protagonista, y deje de ser un simple receptor y consumidor como lo fue en el pasado de valores culturales foráneos. (Cultura, 1982, p. 277).

En sus líneas de acción se enumeró la creación de órganos nacionales de gestión, planificación y administración de la cultura; la organización de eventos culturales que garantizaban "la participación activa y masiva del pueblo", la conservación del patrimonio cultural, el estímulo a la producción de bienes culturales y el fomento al desarrollo de recursos humanos necesarios para este trabajo (Cultura, 1982, p. 278).

Varios experimentos se desarrollaran en los campos de comunicación y cultura, vinculados a esta política cultural del Ministerio, que antes no existían en Nicaragua. Rosario de Mateo (1988) apunta como la política más destacadas del Ministerio, la creación del cine nacional con la fundación del Instituto Nicaragüense de Cine (INCINE); la Agencia de Noticias Nueva Nicaragua (ANN), que mantuvo corresponsales en varios países generando información del país para otros medios de comunicación; Empresa Nicaragüense de Grabaciones Culturales (ENIGRAC), que producía y controlaba la distribución y comercialización de discos y la Editorial Nueva Nicaragua (ENN).

Ernesto Cardenal, en una exposición ante UNESCO en $1982^{2}$ afirmó que la reestructuración de los medios masivos de comunicación fue un ejemplo de la democratización de la cultura sandinista, al reorientar el sistema comunicacional heredado de la dictadura, facilitar el acceso a la comunicación escrita por medio de la alfabetización y la creación de una práctica de discusión pública en programas como "De Cara al Pueblo", del cual profundizaremos adelante. De forma distinta a la política cultural, los pasos propios del campo comunicacional fueron construidos por la revolución sin una sistematización y planificación a medio/largo plazo, en gran parte como consecuencia del contexto de guerra que el país enfrenta desde los primeros años de la revolución.

Una de las primeras acciones en el campo de las comunicación fue la promulgación de la Ley General Provisional sobre los Medios de Comunicación y su reglamento (1979), colocando como pilares centrales el derecho a la información, opinión y expresión, así como la responsabilidad social de los medios de comunicación a favor de los intereses nacionales. En su tercer artículo, la ley señala la obligación del Estado de promover el desarrollo y el progreso de la cultura nicaragüense y la educación, y los medios como vehículos de las mismas. Como contrarios a estos valores se señalaron el uso publicitario de la mujer como objeto sexual, anuncios de bebidas alcohólicas y tabaco, así como la publicación de materiales que "estimulen los vicios, rebajen la dignidad humana o que sean de dañina sensualidad o morbosidad". En el reglamento de la ley merece especial atención el artículo 37, el cual hace mención directa del papel de los medios de comunicación en la construcción de la unidad nacional, con la finalidad de fortalecer y defender los logros de la revolución, la expansión de la educación y la difusión de la cultura nacional, sus principios y tradiciones populares (Nicaragua, 1979).

2 “En la Cultura de Liberación - V Parte". En: Ventana - Barricada Cultural, n 72, 22.5.82, p. 14. 
Entre las diversas experiencias creadas en la reestructuración de los medios de comunicación en Nicaragua, el cambio más grande en términos de estructura organizativa, técnica, política y de lenguaje ocurrió con la televisión (Mateo, 1988). Aunque la radio seguía siendo el medio de mayor capacidad de penetración en el país, la televisión fue incorporada como parte del proyecto de unidad nacional, articulado por los sandinistas de una manera radicalmente diferente a otros medios: un sistema de televisión estatal, el Sistema Sandinista de Televisión (SSTV). Creado a partir de la apropiación de los canales de televisión vinculados a la oligarquía nicaragüense, después de la insurrección popular y la toma del poder, el SSTV asume el Canal 6, propiedad de la familia Somoza, y el Canal 2, propiedad de la familia Sacasa, política y económicamente vinculada a la base de apoyo de la dictadura de Somoza.

El control ideológico somocista en la televisión durante este régimen era directo, $\mathrm{y}$ los dos canales estaban vinculados a la American Broadcasting Co. (ABC), que proporcionaba la mayor parte de los programas y transmitía anuncios de productos industriales estadounidenses (WHEELOCK, 1985). Mario Kaplun (1973) indica que aproximadamente el $50 \%$ de la programación de estos canales era estadounidense y el 25\% de las telenovelas mexicanas (apud MATEO, 1988). La producción de los programas nacionales era mínima y la cobertura de transmisión televisiva se limitaba a las zonas urbanas de la capital Managua y ciudades del Pacífico, como León y Granada, siendo estas regiones más habitadas y viables para la televisión como mercado.
El marco legal de la televisión fue establecido en 1984 como "Corporación Estatal de Derecho Público" y pasó a ser administrada por un Consejo conformado por miembros de los Ministerios de Educación, Cultura, Telecomunicaciones, un representante de la Junta de Gobierno y miembros de la sociedad civil-la asociación nacional de educadores y el sindicato de periodistas - (Nicaragua, 1984). El modelo de televisión adopta el paradigma europeo, proponiendo el monopolio estatal y la finalidad educativa y cultural del medio. Tomas Borge afirmaba en esa época que la adopción de este paradigma estaba relacionada con las nuevas prácticas de comunicaciones directamente enlazadas en el contexto de la transformación social y un amplio espacio para el debate conceptual, que lo convirtió en un modelo típicamente nicaragüense (1988).

Dentro del proceso de estructuración de la televisión sandinista, las principales prioridades fueron, la ampliación de la cobertura a todo el territorio y la promoción de una producción de programas nacionales. Teniendo como institución referente el Ministerio de Cultura, se produjo un fuerte carácter de invención en la producción, con la incorporación de elementos del teatro ${ }^{3} \mathrm{y}$ de la música nacional, así como la creación de géneros nunca antes realizadas en el país, tales como documentales y programas infantiles. La televisión se convierte así en un área prioritaria de inversión pública, incluyendo la importación de receptores ${ }^{4}$. Además de una mayor estructura de transmisión, que alcanzó el $70 \%$ del país en su mejor funcionamiento; se produjo un gran programa de capacitación para

3 Destacados grupos teatrales nicaragüenses como la Compañía de Teatro Justo Rufino Garay y el Teatro de Títeres Guachipilin iniciaron sus actividades en los comienzos del Sistema Sandinista de Televisión.

4 El número de receptores creció de 83 mil en 1975 a 195 mil en 1982. Sin embargo, representaba una relación de apenas 67 receptores por cada mil habitantes, mientras que en EEUU y Canadá llegaba a 660 por mil habitantes. "La radiotelevisión nicaragüense en el contexto mundial de las comunicaciones". En: Ventana - Barricada Cultural, n 224, 15.12.1985, p. 7. 
el personal técnico con la creación de un centro de formación y el establecimiento de acuerdos con numerosos países de América Latina y Europa (O'Donnell, 1993).

En cuanto a su estructura organizativa, el Sistema de Televisión Sandinista estaba compuesto por cuatro áreas: informativa (prensa), administrativa, producción y técnica. Las dos últimas no existían antes de la revolución en la televisión nicaragüense, por la dependencia de los "enlatados" de EE.UU. y México y de la asistencia técnica de estos países con el régimen. La ampliación del acceso a la televisión, sea desde el punto de vista de la creación de nuevos programas como en el hábito de sentirse y verse como "puebloprotagonista", promovió la idea de consolidar la unidad nacional en torno al proyecto de poder sandinista. Sobre todo la creación de programas nacionales con carácter educativo y cultural - que llegaron a ocupar $40 \%$ de la programación - significó el principal logro de la experiencia (Mateo, 1988).

Un reto para la experiencia embrionaria en la televisión, así como para los otros medios de comunicación estatales y/o partidarios del FSLN, era articular sus acciones orgánicamente con diferentes aparatos de la hegemonía de la revolución. Conforme O'Donnell,

...se había detectado la necesidad de pensar los medios de comunicación masiva con relación a otras instancias de producción ideológica de la sociedad, de no separar su función de la función de la institución escolar o la cultura. Dentro del FSLN existía conciencia sobre la necesidad de articular de manera orgánica los diferentes sectores culturales. Se retomaba el concepto de hegemonía de Gramsci, para plantear el propósito de lograr la hegemonía sandinista mediante la unidad nacional (1993, p. 214).

La Cruzada Nacional de Alfabetización - también llamada Insurrección Cultural - es el principal ejemplo de la relación orgánica entre la educación, la cultura y la comunicación sandinistas. Esta organicidad fue uno de los elementos responsables del éxito de la iniciativa, que bajó de manera sustancial los índices de analfabetismo en el país durante el año de 1980. Más de ochenta mil brigadistas, en su mayoría jóvenes de la clase media urbana van al campo, poniéndose en contacto con la pobreza y difíciles condiciones de trabajo de los campesinos. El conflicto entre trabajo manual e intelectual es cuestionado, con la participación mutua de los brigadistas en las actividades productivas rurales y de los trabajadores en las clases de alfabetización. Fuertemente influenciada por la pedagogía de Paulo Freire, la campaña fue cuidadosamente planificada como un "acto político con implicaciones pedagógicas", al movilizar todo el país y promover una comunión del pueblo con su tierra y sus tradiciones (Santos, 1990, p. 77).

La comunicación desempeñó un rol fundamental para el éxito de la campaña. La creación de un sistema de comunicaciones propio, con la participación de los medios masivos estatales, privados, del FSLN y radioaficionados, sustentabalosservicios de información internos y de comunicación de masas, además del "refuerzo las directrices técnicas, pedagógicas y de políticas que se dieron en los talleres de formación". Toda la publicidad y propaganda, incluso en los medios privados, fue totalmente gratuita (Santos, 1990, p. 63).

La televisión, que durante este período tenía un alcance $\mathrm{y} / \mathrm{o}$ recepción exclusivamente urbana, fue utilizada en la etapa preparatoria de la Cruzada para 
transmitir los mensajes de los dirigentes de la Revolución dirigidos a los padres de familia, para que autorizaran a sus hijos e hijas participar en la campaña. Programas culturales como "La Tapisca", de los hermanos Carlos y Luis Enrique Mejía Godoy, y otros promovidos y/o producidos por las organizaciones de masas como "ANDEN", CST, UNAG, hicieron aportes valiosos para el proceso de movilización de toda la población.

Pocos días después de iniciada la campaña alfabetizadora, se creó el programa semanal llamado "Desde la A hasta la Z en la Alfabetización", con mesas redondas, cortos documentales, canciones, mensajes a los alfabetizadores. Un total de 20 ediciones de una hora de duración cada uno se realizaron, "con medios técnicos pequeños y con pocos recursos humanos. Sin embargo su nivel de audiencia era muy alto, dado el gran interés popular por la campaña" ${ }^{5}$.

Debido a la mayor penetración de la radio dentro del territorio nacional, la estrategia fue distinta. Antes de iniciada la campaña se realizó el programa "La Insurrección Cultural en Marcha", para motivar la participación del campesino en el proceso de alfabetización así como de cuñas publicitarias del Censo Nacional de Alfabetización. Pero el logro más grande de todo el esfuerzo radial fue el programa "Puño en Alto", emitido en dos ediciones diarias de 45 minutos en cadena nacional.

Concebido en sus inicios como un servicio a la alfabetización, el programa "Puño en Alto" cambia de manera gradual su contenido a apoyo técnico y pedagógico de los brigadistas, integrando en el programa temas como salud, servicio social, información periodística, orientaciones pedagógicas, etc. Losperiodistas, trabajando de manera voluntaria, transmitían desde las regiones donde escenificaba la Cruzada, socializando las condiciones de trabajo y los retos que enfrentaban los brigadistas en cada localidad. "Puño en Alto" llegó a ser el programa radial más escuchado en Nicaragua durante la Cruzada, siendo un medio de estímulo, orientación y primordialmente de unidad de la campaña (Educación, 1981).

En resumen, por medio de la comunicación, se fortaleció el valor de la educación y la cultura, habiendo una reducción del analfabetismo estructural en la sociedad nicaragüense, el cual llegaba a casi el 100\% de las mujeres rurales. En cinco meses que duró la campaña, más de 400.000 nicaragüenses fueron alfabetizados. La tarea de enfrentar las distancias geográficas, romper con las barreras ancestrales, económicas, étnicas, sociales y políticas de las "diferentes" Nicaragua, existente en el país, en un intento de unidad nacional por medio de la cultura, movilizó a todos los grupos sociales, en especial a su juventud alrededor de la Cruzada.

En cuanto a los medios de comunicación, es cierto que la labor desempeñada por cada uno de ellos fue fundamental para el éxito de la campaña. Sin embargo, "hay que señalar que el principal vehículo de comunicación ha sido el propio pueblo, que con su entusiasmo contagioso fue a la montaña y desde las ciudades como retaguardia, apoyó a los muchachos y muchachas brigadistas" ${ }^{\prime \prime}$.

5 Conforme el documento del "I Congreso de la Cruzada Nacional de Alfabetización", 1980, p. 25. Acervo del Instituto de Historia de Nicaragua y Centroamérica, Universidad Centroamericana.

6 Documento del "I Congreso de la Cruzada Nacional de Alfabetización", 1980, p. 30. Acervo del Instituto de Historia de Nicaragua y Centroamérica, Universidad Centroamericana. 
Otra experiencia destacada de la comunicación sandinista fue el programa "De Cara al Pueblo", creado para ser un espacio de diálogo permanente entre la Junta de Gobierno de Reconstrucción Nacional (JGRN) y la Dirección nacional del FSLN con el pueblo. A partir de encuentros semanales con diferentes sectores sociales y desde una diversidad de lugares del país, el programa discutía la coyuntura del momento que pasaba la revolución, las políticas implementadas dentro de ese difícil contexto y atendía las demandas específicas del sector o localidad invitada. El protagonismo popular se manifestaba en las críticas constructivas del pueblo a los dirigentes, debatiendo sobre los principales problemas del pueblo y en la beligerancia de las organizaciones de masas invitadas a cada encuentro.

Transmitido por el Sistema Sandinista de Televisión y por la radio $\mathrm{La} \mathrm{Voz} \mathrm{de}$ Nicaragua, el programa era denominado en su inicios de apertura como "síntesis de la democracia popular sandinista" y gradualmente se tornó en "el programa propagandístico más importante de la Revolución"7. Este programa, junto a otros de tipo radiales como "Contacto 6.20" y "Línea Directa", fueron interesantes experimentos en comunicación dialógica de raigambre popular al mezclar dentro del espacio de transmisión la crítica popular con el análisis amplio y la discusión en vivo sobre los desafíos y limitaciones del proceso sandinista.

La revolución nicaragüense no se perdió en el escenario mundial. Si bien era un país pequeño, poco conocido y mal integrado en el capitalismo global, rápidamente movilizó las potencias extranjeras en un embargo contrarrevolucionario. Los diez años del FSLN en el gobierno fueron constantemente amenazados por una "guerra de baja intensidad", financiada por los Estados Unidos, cuyo objetivo era desestabilizar el país y destruir su economía (Escalante, 2008). Los medios de comunicación hicieron parte de esa guerra: había una deliberada contrapropaganda que incluyó la penetración de señales de radio y televisión de otros países en Nicaragua. Rosario de Mateo señala que, en 1985, llegaron a Nicaragua "las emisiones de 15 televisoras extranjeras, principalmente de Honduras, Costa Rica y Estados Unidos, afectando a todo el país, pero primordialmente a las zonas fronterizas donde las necesidades provocadas por la guerra eran más acuciantes" (Mateo, 1988, p. 94). Como mecanismo de defensa en un contexto de guerra, los sandinistas establecen como institución rectora de los medios de comunicación al Ministerio de Interior (MINT), estructurando de manera rápida un criterio de atención y/o supervisión a partir del rol político de los medios en la lucha ideológica y militar que se libraba.

Con la derrota electoral del FSLN en las elecciones de 1990, donde sale triunfante la coalición opositora UNO encabezada por doña Violeta Barrios de Chamorro, se produce el proceso de desmantelamiento del marco jurídico para la comunicación establecido por el gobierno revolucionario. Rothschuh Villanueva afirmó que con esta nueva situación se perdió un modelo que promovía la participación de los diversos sectores sociales, en un intento por democratizar la comunicación en Nicaragua (1992). El gobierno de "Doña Violeta" (como popularmente le llamaban) reduce de manera brusca el presupuesto del Sistema Nacional de Televisión,

7 "A un año de su inicio... De Cara al Pueblo: ejemplo de verdadera democracia popular". En: Barricada, n 660, 6.6.1981, p. 4. 
conformado únicamente por el Canal 6, ya que el 2 fue regresado a la familia Sacasa, dentro de un proceso draconiano de privatización y de reducción del aparato estatal.

Durante la transición de gobierno el FSLN se asigna la frecuencia del canal 4, la cual mantiene hasta la actualidad. Dentro de la televisión estatal, los primeros pasos dado por el gobierno neoliberal de Doña Violeta se manifiestan en el despido masivo de los trabajadores vinculados al FSLN y la destrucción de la mayor parte de la colección audiovisual que documentaba los logros del período sandinista.

A pesar de la singularidad de la experiencia nicaragüense en las políticas de comunicación y cultura, especialmente en la construcción de su televisión estatal, es interesante analizar la experiencia a partir del establecimiento de un diálogo con las nuevas orientaciones de las políticas públicas de comunicación en América Latina. El debate sobre la acción del Estado en el campo de los medios de comunicación como disputa por la hegemonía permanece encendido en el contexto actual de nuestro continente. Incluso el contexto de la "guerra de baja intensidad" ocurrida en Nicaragua puede relacionarse con los intentos de golpe a la institucionalidad que países como Venezuela, Ecuador, Bolivia, Honduras y Paraguay han sufrido recientemente. Eventos que por cierto afectan la conformación de los medios de comunicación del Estado y la consolidación de las políticas públicas de comunicación.

\section{Consideraciones Finales}

La experiencia de Nicaragua es muy instructiva, principalmente en tres aspectos. El primero, porque reúne en un solo esfuerzo, la comunicación y la cultura política. Los medios de comunicación se convierten en sujetos de la erradicación del analfabetismo y la recuperación de las identidades culturales olvidadas y borradas. No hay una oposición entre una comunicación que entretiene y una cultura que educa. La comunicación también se asume como cultura, riesgos y responsabilidades que vienen de esa opción.

El segundo aspecto es la posibilidad de reflexionar sobre el rol de los medios de comunicación, más allá de la lógica del mercado, que comúnmente los vinculan en América Latina. Medios de comunicación para un mundo en que la transformación social y el consumo no sean sinónimos, ni que la primera esté subordinada al segundo.

Por último, Nicaragua nos enseña que es posible pensar en una identidad nacional como forma de resistencia y como parte de un proyecto popular de poder. En otras palabras, enseña a tratar la identidad nacional no como un concepto monolítico, cuyo costo es la aniquilación de las diferencias, sino plural donde se tome en cuenta los conflictos entre grupos, entre lenguas, entre etnias y que aporte a la soberanía de su pueblo. 


\section{Referencias Bibliográficas}

BORGE, Tomas. Incorporemos al pueblo a la comunicación. Managua: Centro de Capacitación de CORADEP (Corporación de Radio Difusión del Pueblo), 1988.

BRASIL. Ministério da Cultura. As Metas do Plano Nacional de Cultura. São Paulo: Instituto Via Pública; Brasília: MinC, 2012.

BARBALHO, Alexandre. Políticas de cultura, políticas de identidade. XXIV Congresso Brasileiro de Ciências da Comunicação - Campo Grande - MS, 2001. En: < http:// www. portcom.intercom.org.br/pdfs /3125832307473113147671051549207 41375 72.pdf $>$. Acceso en 15 de junio de 2013.

BRITTOS, Valério Cruz; MIGUEL, João. Políticas de comunicação e sociedade civil: implicações conceituais e desafios práticos. VIII Congresso de Ciências da Comunicação na Região Norte - Porto Velho - RO, 2009. En: < http://www.intercom.org. br /papers/ regionais/norte2009/ resumos/R18-0128-1.pdf>. Acceso en 15 de junio de 2013.

CULTURA, Ministerio de. Hacia una política cultural de la Revolución Popular Sandinista. Managua: Ministerio de Cultura, 1982.

EDUCACIÓN, Ministerio de. Nicaragua triunfa en la alfabetización: documentos y testimonios de la Cruzada Nacional de Alfabetización. San José: DEI, 1981.

ESCALANTE, Fabián. Operación Calipso. La guerra sucia de los Estados
Unidos contra Nicaragua, 1979-1983. México D.F.: Ocean Sur, 2008.

KEHL, Maria Rita. Eu vi um Brasil na TV. In: I. F. SIMÕES, A. H. da COSTA, M. R. KEHL (Orgs.). Um país no ar: história da TV brasileira em três canais. São Paulo: Brasiliense, 1986.

MATEO, Rosario de. Poder y modelo de comunicación em Nicarágua: de Somoza García al sandinismo. Revista CIDOB d'afers internacionals, n. 14-15, Barcelona, 1988, pg. 81-99. En: < http://www. raco.cat/index.php/revistacidob/ article/viewFile/27793/51920>. Acceso en 15 de junio de 2013.

NICARAGUA. Dirección de divulgación y Prensa. Ley General y Reglamento sobre los medios de comunicación. Managua: Patria Libre, 1979.

NICARAGUA, Junta de Gobierno de Reconstrucción Nacional de la República de. Ley de Creación del Sistema Sandinista de Televisión. Decreto No. 1398 de 10 de febrero de 1984. La Gaceta, n.38, de 22 de febrero de 1984. En: <http://legislacion. asamblea.gob.ni/ normaweb. nsf/9e314815a08d4a6206257265005d 21f9/8c96e58e996707e5062570a1005 70a10057c271?OpenDocument>. Acceso en 15 de junio de 2013.

O'DONNELL, Penelope Ann. La enseñanza-aprendizaje de la comunicación en Nicaragua durante la Revolución Popular Sandinista. Tesis de maestría en comunicación - Universidad Ibero-Americana. México, DF, 1993.

ORTIZ, Renato. A moderna tradição brasileira. São Paulo: Brasiliense, 1994. $5^{\text {a }}$ ed. 
Cultura brasileira e identidade nacional. São Paulo: Brasiliense, 2003. $5^{\mathrm{a}}$ ed.

Diversidade cultural e cosmopolitismo. Lua Nova, n. 47, São Paulo, 1999, p. 73-89.

RAMA, Ángel. Transculturación narrativa en América Latina. México: Siglo XXI editores, 2004.

SANTOS, Nilton. E também lhes ensinem a ler...: a experiência da Cruzada Nacional de Alfabetização da Nicarágua. Rio de Janeiro: Ayuri Editorial, 1990.

VILLANUEVA, Guillermo Rothschuh. Cambio de carril. Managua: Universidad Centro-Americana, 1992.

WHEELOCK ROMÁN, Jaime. Imperialismo y dictadura. Managua: Nueva Nicaragua, 2570a10057c271?OpenDocument>. Acceso en 15 de junio de 2013. 